\title{
Challenges in the diagnosis and treatment of small fiber neuropathies
}

\author{
Desafios no diagnóstico e tratamento das neuropatias de fibras finas
}

Josep Valls-Solé1

\author{
Universitat de Barcelona, Facultat de \\ Medicina, Hospital Clinic, Neurology \\ Department, EMG Unit, Institut \\ d'Investigació Biomèdica August Pi i \\ Sunyer, Barcelona, Spain.

\section{Correspondence:} \\ Josep Valls-Sole; Unitat \\ d'Electromiografia. Servei de \\ Neurologia. Villarroel, 170. Hospital \\ Clínic, Barcelona, Spain. E-mail: \\ jvalls@clinic.ub.es \\ Conflict of interest: \\ There is no conflict of interest to \\ declare. \\ Received 16 February 2018 \\ Accepted 19 February 2018.
}

\section{(c) $\mathbf{B Y}$}

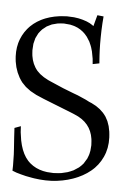

mall fiber neuropathies (SFN) are challenging at the level of the diagnosis, treatment and follow-up. Patients usually complain of severe pain, mostly in their feet, greatly affecting their quality of life, while the examiner finds no, or very few, clinical signs of peripheral neuropathy. Conventional electrodiagnostic testing does not give much additional information and, in idiopathic cases, routine blood tests do not show any abnormality. What to do in these cases? Symptomatic treatment is also often disappointing. Antiepileptic drugs, serotonin reuptake inhibitors and tricyclic antidepressants are the first line of treatment ${ }^{1}$. They may improve $30 \%$ in the rating of pain scores in about $30 \%$ of the patients, which is not much different from the effect of placebo. Being a little more aggressive with pharmacological treatment leads to opioids, which may produce side effects, and invasive treatments such as neural blockade, spinal cord stimulation, intrathecal medication, and neurosurgical interventions, which are elected by some patients impelled by the severity of their symptoms.

On this topic, as in many others, a correct diagnosis is more than half the medical action to be taken. A thorough explanation to the patient about the nature of the problem, the origin of his/her symptoms, the benefits and side effects of the available medication and the reassurance of close follow-up are the most logical steps to complete the medical act. However, symptoms may continue in spite of all the care. It is in this situation that we need guidelines and consensus papers, like that by Gondim et al., in this issue ${ }^{2}$. Experience from other experts in the field becomes very handy when we, and our patients, need reassurance in the diagnosis. In fact, the syndromic diagnosis of SFN is not a difficult one to reach on the basis of clinical assessment. It is more difficult to have laboratory support of the diagnosis, as conventional electrodiagnostic methods are insufficient and more sophisticated equipment is not accessible to all ${ }^{3}$. It is even more difficult to reach an etiological diagnosis. The percentage of unknown causes of SFN remains at about 30\% of the patients, in spite of advancement in the tools used for the diagnosis. ${ }^{4}$. In those with known causes, impaired glucose metabolism, chronic inflammatory demyelinating polyneuropathy, and monoclonal gammopathies are the disorders most frequently observed. The importance of a complete blood test for an etiological diagnosis has been stressed by Lang et al. ${ }^{5}$. These authors determined the prevalence of each abnormal blood test result among a battery of 21 tests. The most prevalent abnormalities were a high erythrocyte sedimentation rate and antinuclear antibodies, each present in $28 \%$ of 231 patients with skin biopsy confirmation of SFN. Apart from that, a number of other antibodies with elevated titers were found, which led the authors to suggest an association between SFN and dysimmunity.

As Gondim et al. ${ }^{2}$ correctly point out in their manuscript, the use of relatively sophisticated electrodiagnostic techniques has led us to uncover a larger number of patients with SFN. Electrodiagnostic testing has an important task in the characterization of the nerve lesion causing SFN. Probably the closest method to examine conduction in small fibers is microneurography, which may help in identifying the type of sensory fibers involved and the severity of the nerve lesion ${ }^{6}$. However, pain is a feeling and characterization of the lesion that may not take into account all the mechanisms involved. A useful method for the evaluation of how patients feel pain is psychophysical testing, i.e., quantitative sensory testing. Small fibers convey temperature and pain sensations and it is, therefore, relevant to assess thermoalgesic sensation using 
psychophysical methods in SFN patients. A rather complex protocol for quantitative sensory testing has been promoted by the German Research Network on Neuropathic Pain for the description of sensory profiles of different forms of polyneurop$a^{2}{ }^{7}$. Another proposal has been to examine how patients feel temperature change with the so-called dynamic thermotest ${ }^{8}$.
In any case, the systematic use of the available electrodiagnostic and quantitative sensory testing tools helps us to describe more and more forms of clinical manifestations of SFN ${ }^{9,10}$ and their pathophysiological mechanisms. This will eventually lead to recognition of biomarkers of the dysfunction and provide cues to devise new treatments.

\section{References}

1. Dworkin RH, O'Connor AB, Audette J, Baron R, Gourlay GK, Haanpää $\mathrm{ML}$ et al. Recommendations for the pharmacological management of neuropathic pain: an overview and literature update. Mayo Clin Proc. 2010 Mar;85(3 suppl):S3-14. https://doi.org/10.4065/mcp.2009.0649

2. Gondim FAA, Barreira AA, Claudino R, Cruz MW, Cunha FMB, Freitas $M R G$ et al. Definition and diagnosis of small fiber neuropathy: consensus from the Peripheral Neuropathy Scientific Department of the Brazilian Academy of Neurology. Arq Neurpsiquiatr 2018;76(3):189-97. https://doi.org/10.1590/0004-282X20180015

3. Barraza-Sandoval G, Casanova-Mollá J, Valls-SoléJ. Neurophysiological assessment of painful neuropathies. Expert Rev Neurother. 2012 Nov;12(11):1297-309. https://doi.org/10.1586/ern.12.93

4. Farhad K, Traub R, Ruzhansky KM, Brannagan TH 3rd. Causes of neuropathy in patients referred as "idiopathic neuropathy". Muscle Nerve. 2016 Jun;53(6):856-861. https://doi.org/10.1002/mus.24969

5. Lang M, Treister R, Oaklander AL. Diagnostic value of blood tests for occult causes of initially idiopathic smallfiber polyneuropathy. J Neurol. 2016 Dec;263(12):2515-27. https://doi.org/10.1007/s00415-016-8270-5
6. Serra J. Microneurography: towards a biomarker of spontaneous pain. Pain. 2012 Oct;153(10):1989-90. https://doi.org/10.1016/j.pain.2012.07.008

7. Rolke R, Baron R, Maier C, Tölle TR, Treede RD, Beyer A et al. Quantitative sensory testing in the German Research Network on Neuropathic Pain (DFNS): standardized protocol and reference values. Pain. 2006 Aug;123(3):231-43. https://doi.org/10.1016/j.pain.2006.01.041

8. Medici C, Barraza G, Castillo CD, Morales M, Schestatsky P, Casanova-Mollà Jet al. Disturbed sensory perception of changes in thermoalgesic stimuli in patients with small fiber neuropathies. Pain. 2013 Oct;154:2100-7. https://doi.org/10.1016/j.pain.2013.06.034

9. Serra J, Solà R, Quiles C, Casanova-Molla J, Pascual V, Bostock H et al. C-nociceptors sensitized to cold in a patient with small-fiber neuropathy and cold allodynia. Pain. 2009 Dec;147(1-3):46-53. https://doi.org/10.1016/j.pain.2009.07.028

10. Skorna M, Kopacik R, Vlckova E, Adamova B, Kostalova M, Bednarik J. Small-nerve-fiber pathology in critical illness documented by serial skin biopsies. Muscle Nerve. 2015 Jul;52(1):28-33. https://doi.org/10.1002/mus.24489 